\title{
VIBRATIONS CONTROL OF THE HARMONICALLY EXCITED NONLINEAR SYSTEM VIA POSITIVE POSITION FEEDBACK CONTROLLER
}

\author{
Y. A. Amer a , A. T. EL-Sayed ${ }^{\text {b }}$, A. A. Saleh ${ }^{\text {c }}$, A. M. Abdel-Wahab ${ }^{\text {c }}$ H. F. Salman ${ }^{\text {d, * }}$ \\ ${ }^{a}$ Department of Mathematics, Faculty of Science, Zagazig University, Zagazig, Egypt \\ ${ }^{b}$ Department of Basic Sciences, Modern Academy for Engineering and Technology, Maadi, Egypt \\ ${ }^{c}$ Department of Mathematics, Faculty of Science(Girls), Al-Azhar University, Nasr City, Egypt \\ ${ }^{d}$ Department of Basic Sciences, Faculty of Computers and Information, Cairo University, Giza, Egypt \\ *Email: halasalman35@yahoo.com (H.F. Salman)
}

\begin{abstract}
In this paper, the vibration reducing of the harmonically excited nonlinear system is presented via applying positive position feedback controller (PPF). The analytical results are obtained by applying the multiple-scale perturbation techniques (MSPT) up to second-order approximations. The frequency response equation (FRE) is studied to test the behavior of the steady state solutions near the simultaneous resonances. The effects of the several parameters and the behavior of the system at resonance case are investigated to illustrate the optimum working conditions for the PPF controller. The stability analysis for the uncontrolled system is investigated by applying the phase portrait technique. Moreover, numerical simulation is used to compare between time-history and the analytical solution. The analytical and numerical solutions are compared to show the validity of the results. Finally, a comparison with the available results in the literature is presented.
\end{abstract}

\section{Keywords}

Nonlinear system; Multiple scales method; Resonance; PPF controller; Stability, phase plane.

\section{INTRODUCTION}

The study of nonlinear systems is of great importance in physics, since physical phenomena in nature are intrinsically nonlinear. Therefore, here is a challenge in various fields of research to understand the nonlinear systems such that we can develop novel approaches depend in the theory of dynamical modeling [1]. There is extensive research work studying the solution of such nonlinear systems in literature. For instance, Ref. [2], examined combining the pitch and roll motion of ships. Hence, the saturation phenomenon was discovered. Since, the design passive vibration control is too complex and difficult to control exactly, the percentage of internal resonance and active vibration control has been used. Therefore, many authors implement advanced control.

In the work of Ref. [3] the horizontal oscillation of the magnetic moving body object has been reduced by the including a nonlinear saturation controller (NSC) to the nonlinear differential equation. Multivariate the technique of multiple scales was applied to get an analytical solution and to explain the behavior of this model. In another research paper [4] loop delays have been taken into consideration, when PPF controller was applied. In that case, vibrations of self-excited and forced nonlinear beam could be reduced and approximate solution was studied after using MTSP technique. On the other hand, analytical formulas for calculating the induced resonance frequency transformations of electrically charged, micro-and nano-imposed (carbon nanotubes) beams, were obtained [5], due to adding mass. The linear dynamical responses of the added masses were investigated after being systemized on a separate point of masses that depend in the theory of Euler Bernoulli beam. And finally a comparison was made between the analytical results and the numerical solution to the problem. Moreover, the beam can be cracked and with the influence of harmonic excitement, using different control techniques [6]. These controls are Positive position feedback (PPF), integral resonance control (IRC) and nonlinearity NIPPF observations. To obtain the mathematical analysis and observing the curves of multi-branch frequency amplitude, multiple scales process was applied. Additionally, the sensitivity of the frequency responses to the frequency ratio 
increased in higher depth ratios. The nonlinear s.d.o.f. system of the fifth order under external stirring with two distinct delay time, was studied in [7]. They introduced an investigation of primary and super harmonic of order five, and subharmonic of order one-three resonances for this system. The equations of the modulation for the amplitudes and the phases have determined by using the technique of multiple scales. They also investigated in each resonance about the stabilities regions and their steady-state solutions. In addition, by using the Mathematica Software, the numerical results were obtained. Finally they discussed the influence of the time-delays and feedback gains on the non-linear response of this system. To reduce the peak amplitude of the response for the case of primary, they have chosen a suitable time-delays and feedback gains that could increase the critical force amplitude.

The stability of the non-autonomous bicircular four-body system, has been analyzed in [8]. By using Newton's law of gravity they derived the governing equation. In Ref. [9], the multiple scales perturbation method was used to check oscillations in the compressor rotating blade. In order to decrease the oscillations in this model, they applied position with velocity feedback controller. In Ref. [10] mass presented analytical formulations was added in order to compute the persuaded resonance frequency shifts of electrically activated clamped-clamped micro and nano (Carbon nanotube) beams. In this case, the beam's linear dynamic responses added masses depend on the theory of EulerBernoulli beam. The oscillations of a self-excited and forced nonlinear beam applied PPF controller has been reduced in [11]. MSPT has been used to obtain an approximate solution of first-order in the simultaneous resonance case. The curves of the equilibrium solution are designed for various values of controller parameters. They investigated the steady state solution by using the equations of frequency-response. Then the approximate solution was verified numerically.

In Ref. [12], stochastic dynamic of a piezoelectric energy harvesting device, was studied. They presented the lumped parameter of physical model representing the device, hence they developed a normalized mathematical system for simulating the model. Accordingly, they applied the random vibration theory in order to describe the device's output power in terms of the excitation of the neighboring media. Then, they have maximized the mean value of the normalized gathered power. On the other hand, the multi Positive Feedback (MPF) control was studied in [13]. That was a new technique for active vibration reduction in flexible smart constructions. The same authors [14] studied a novel Nonlinear Integral Resonant Controller (NIRC). In that respect, the vibrations in nonlinear vibrational smart structures were suppressed using the method of Multiple Scales. In that case, they could get an analytical solution for the closed-loop model. The multi-body dynamical system of the Virginia Tech Roller Rig (VTRR) was developed [15], via multi body simulation software package SIMPACK. They used multi body dynamical system for conducting vibration, noise, harshness analysis of the rig. In this regard, the Integral Consensus Control (ICC) can be introduced [16], as a novel vibration control procedure. Therefore, the unwanted oscillations in flexible structures can be suppressed. The absorber methodology was a network-based distributed technique, and used consensus control design. However, the vibration of a nonlinear model can be [17], using PPF active controller, when subjected to external primary resonance excitation and one can obtain a first-order analytical solution using MSPT.

Nonlinear oscillations of flexible structures can be reduced [18], using nonlinear modified positive position feedback (NMPPF). Where a PPF controller was modified by a first-order compensator. In Ref. [19] the PPF absorber was considered to reduce the oscillations of a vertical conveyor. Here, two PPF controllers were used to reduce the vertical vibration in a vertical conveyor. In this respect, two PPF controllers can be integrated on a system of horizontally Jeffcott-rotor, in order to suppress the vertical and horizontal vibrations of this system. Therefore, one can obtain the analytical solution of a second-order governing equations of system that includes cubic and quadratic nonlinear terms. by applying MSPT. In a nutshell, the previous studies was considered to reduce the vibrations of the various dynamic models by adding controllers such as PPF, NSC, NIPPF and etc. or by studying the delay of time. However, in this article, we investigate an active vibration control with external excitations of a nonlinear s.d.o.f. system of the fifth order mentioned in [7] by applying positive position feedback (PPF) controller. 
The article is organized as follows: in Sec. (2), we study the Equations of the modeling system. Then, multiple timescales perturbation (MTSP) technique is used to obtain a second-order analytical solution. And, the stability of the steady-state solution was examined by using the equations of frequency-response. Moreover, the stability of the steady-state solution is examined by using the equations of frequency-response. In Sec. (3), to explain the stability classifications the method of phase portrait is introduced. Sec. (4) is devoted to show discussions and Results. The curves of equilibrium solution were plotted for several values of absorber parameters. In addition, we noticed that all predictions from approximate solutions are in good validity with the simulation from the numerical solutions. We do a comparison between our results and other results in the literature as in $\mathrm{Sec}$. (5). At the end in Sec. (6) we give our conclusions.

\section{Equations of the modeling system}

In this paper, the main objective is to investigate the non-linear dynamical behavior of a harmonically excited non-linear single-degree-of-freedom (s.d.o.f.) model as in [7] after adding PPF controller.

$$
\begin{aligned}
& \ddot{x}+\omega_{1}^{2} x+\left(2 \mu_{1} \dot{x}+\alpha_{1} x^{2}+\alpha_{2} x^{3}+\alpha_{3} x^{4}+\alpha_{4} x^{5}\right)=F \operatorname{Cos}(\Omega t)+\gamma y, \\
& \ddot{y}+\omega_{2}^{2} y+2 \mu_{2} \dot{y}=\lambda x,
\end{aligned}
$$

Where $\omega_{1}$ and $\omega_{2}$ are linear natural frequencies of main system and controller, respectively, $\mu_{1}$ and $\mu_{2}$ are the damping coefficients of main system and controller are, respectively, $\alpha_{1}, \alpha_{2}, \alpha_{3}$ and $\alpha_{4}$ are the nonlinear parameters of the main system, $\gamma$ is the Feedback signal gain, $\lambda$ is the Control signal gain.

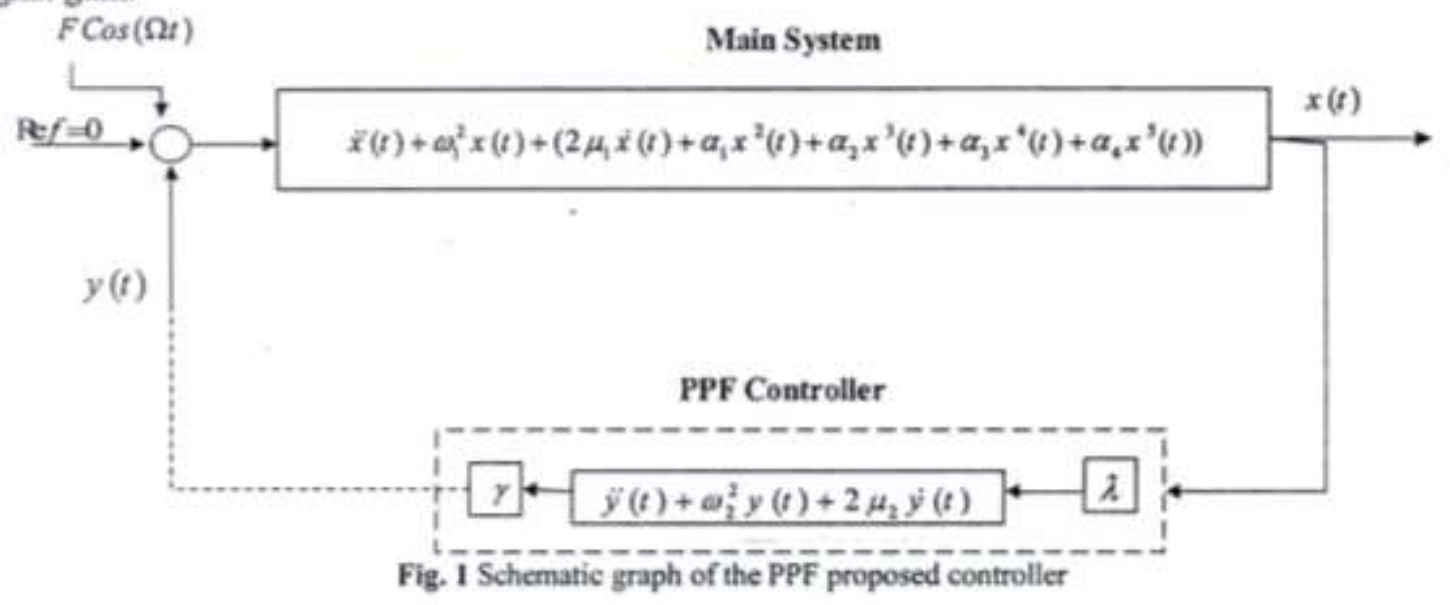

Using the perturbation technique MSPT as follow:

$$
\begin{aligned}
& x(t ; \varepsilon)=x_{0}\left(T_{0}, T_{1}, T_{2}\right)+\varepsilon x_{1}\left(T_{0}, T_{1}, T_{2}\right)+\varepsilon^{2} x_{2}\left(T_{0}, T_{1}, T_{2}\right), \\
& y(t ; \varepsilon)=y_{0}\left(T_{0}, T_{1}, T_{2}\right)+\varepsilon y_{1}\left(T_{0}, T_{1}, T_{2}\right)+\varepsilon^{2} y_{2}\left(T_{0}, T_{1}, T_{2}\right),
\end{aligned}
$$

The time derivatives can be established as follows:

$$
\begin{aligned}
& \frac{d}{d t}=D_{0}+\varepsilon D_{1}+\varepsilon^{2} D_{2}, \\
& \frac{d^{2}}{d t^{2}}=D_{0}^{2}+2 \varepsilon D_{0} D_{1}+\varepsilon^{2}\left(D_{1}^{2}+2 D_{0} D_{2}\right),
\end{aligned}
$$


Where $D_{r}=\partial / \partial T_{r}, r=0,1,2$.

One can scale the parameters of Eqs. (1) and (2) as follows

$\mu_{1}=\varepsilon^{2} \hat{\mu}_{1}, \mu_{2}=\varepsilon^{2} \hat{\mu}_{2}, F=\varepsilon \hat{F}, \alpha_{i}=\varepsilon \hat{\alpha}_{i}(i=1,2,3,4), \gamma=\varepsilon \hat{\gamma}, \lambda=\varepsilon \hat{\lambda}$.

Substituting Equations (3) - (6) into Equations (1)- (2), then we equate the same powers for $\varepsilon$ of the coefficients resulting equations in the following:

$\operatorname{Order}\left(\varepsilon^{0}\right)$ :

$$
\begin{aligned}
& \left(D_{0}^{2}+\omega_{1}^{2}\right) x_{0}=0 \\
& \left(D_{0}^{2}+\omega_{2}^{2}\right) y_{0}=0
\end{aligned}
$$

$\operatorname{Order}(\varepsilon)$ :

$$
\begin{aligned}
& \left(D_{0}^{2}+\omega_{1}^{2}\right) x_{1}=-2 D_{0} D_{1} x_{0}-\hat{\alpha}_{1} x_{0}^{2}-\hat{\alpha}_{2} x_{0}^{3}-\hat{\alpha}_{3} x_{0}^{4}-\hat{\alpha}_{4} x_{0}^{5}-2 \hat{\mu}_{1} D_{0} x_{0}+\hat{F} \operatorname{Cos}(\Omega t)+\hat{\gamma} y_{0} \\
& \left(D_{0}^{2}+\omega_{2}^{2}\right) y_{1}=-2 D_{0} D_{1} y_{0}-2 \hat{\mu}_{2} D_{0} y_{0}+\hat{\lambda} x_{0}
\end{aligned}
$$

Order $\left(\varepsilon^{2}\right)$ :

$$
\begin{aligned}
\left(D_{0}^{2}+\omega_{1}^{2}\right) x_{2}= & -D_{1}^{2} x_{0}-2 D_{0} D_{1} x_{1}-2 D_{0} D_{2} x_{0}-2 \hat{\mu}_{1}\left(D_{0} x_{1}+D_{1} x_{0}\right)-2 \hat{\alpha}_{1} x_{0} x_{1}-3 \hat{\alpha}_{2} x_{0}^{2} x_{1} \\
& -4 \hat{\alpha}_{3} x_{0}^{3} x_{1}-5 \hat{\alpha}_{4} x_{0}^{4} x_{1}+\hat{\gamma} y_{1} \\
\left(D_{0}^{2}+\omega_{2}^{2}\right) y_{2}= & -D_{1}^{2} y_{0}-2 D_{0} D_{1} y_{1}-2 D_{0} D_{2} y_{0}-2 \hat{\mu}_{2}\left(D_{0} y_{1}+D_{1} y_{0}\right)+\hat{\lambda} x_{1}
\end{aligned}
$$

The solution of Eqs. (7) - (8), can be expressed in the form

$$
\begin{aligned}
& x_{0}=A\left(T_{1}, T_{2}\right) e^{i \omega_{0} T_{0}}+c c \\
& y_{0}=B\left(T_{1}, T_{2}\right) e^{i \omega_{2} T_{0}}+c c
\end{aligned}
$$

Substituting Eqs. (13) - (14) into Eqs. (9)- (10), we get the following:

$$
\begin{gathered}
\left(D_{0}^{2}+\omega_{1}^{2}\right) x_{1}=\hat{\gamma} B \exp \left(i \omega_{2} T_{0}\right)-\left(\hat{\alpha}_{1} A^{2}+4 \hat{\alpha}_{3} A^{3} \bar{A}\right) \exp \left(2 i \omega_{1} T_{0}\right)-\hat{\alpha}_{3} A^{4} \exp \left(4 i \omega_{1} T_{0}\right) \\
-\left(\hat{\alpha}_{2} A^{3}+5 \hat{\alpha}_{4} A^{4} \bar{A}\right) \exp \left(3 i \omega_{1} T_{0}\right)-\hat{\alpha}_{4} A^{5} \exp \left(5 i \omega_{1} T_{0}\right)+\frac{1}{2} \hat{F} \exp \left(i \Omega T_{0}\right) \\
-\left(3 \hat{\alpha}_{2} A^{2} \bar{A}+10 \hat{\alpha}_{4} A^{3} \bar{A}^{2}+2 i \hat{\mu}_{1} \omega_{1} A+2 i \omega_{1} D_{1} A\right) \exp \left(i \omega_{1} T_{0}\right) \\
-\left(\hat{\alpha}_{1} A \bar{A}+3 \hat{\alpha}_{3} A^{2} \bar{A}^{2}\right)+c c . \\
\left(D_{0}^{2}+\omega_{2}^{2}\right) y_{1}=-\left(2 i \hat{\mu}_{2} \omega_{2} B+2 i \omega_{2} D_{1} B\right) \exp \left(i \omega_{2} T_{0}\right)+\hat{\lambda} A \exp \left(i \omega_{1} T_{0}\right)+c c .
\end{gathered}
$$

The solutions of Eqs. (15)- (16) after eliminating secular terms are

$$
\begin{aligned}
x_{1}= & C_{1}\left(T_{1}, T_{2}\right) \exp \left(i \omega_{2} T_{0}\right)+C_{2}\left(T_{1}, T_{2}\right) \exp \left(2 i \omega_{1} T_{0}\right)+C_{3}\left(T_{1}, T_{2}\right) \exp \left(3 i \omega_{1} T_{0}\right)+C_{4}\left(T_{1}, T_{2}\right) \\
& \times \exp \left(4 i \omega_{1} T_{0}\right)+C_{5}\left(T_{1}, T_{2}\right) \exp \left(5 i \omega_{1} T_{0}\right)+C_{6}\left(T_{1}, T_{2}\right) \exp \left(i \Omega T_{0}\right)+C_{7}\left(T_{1}, T_{2}\right)+c c . \\
y_{1}= & C_{8}\left(T_{1}, T_{2}\right) \exp \left(i \omega_{1} T_{0}\right)+c c .
\end{aligned}
$$


where $C_{j}(j=1,2, \ldots, 8)$ are complex functions in $T_{1}$ and $T_{2}$, which are presented in the appendix.

Similarly, by substituting from Eqs. (17) - (18) into Eqs. (11)- (12), then exclusion the secular terms from equations (19), (20), the general solutions of these equations are obtained as follows

$$
\begin{aligned}
x_{2}= & E_{1}\left(T_{1}, T_{2}\right) \exp \left(9 i \omega_{1} T_{0}\right)+E_{2}\left(T_{1}, T_{2}\right) \exp \left(8 i \omega_{1} T_{0}\right)+E_{3}\left(T_{1}, T_{2}\right) \exp \left(6 i \omega_{1} T_{0}\right)+E_{4}\left(T_{1}, T_{2}\right) \\
& \times \exp \left(7 i \omega_{1} T_{0}\right)+E_{5}\left(T_{1}, T_{2}\right) \exp \left(i \omega_{2} T_{0}\right)+E_{6}\left(T_{1}, T_{2}\right) \exp \left(2 i \omega_{1} T_{0}\right)+E_{7}\left(T_{1}, T_{2}\right) \exp \left(i\left(2 \omega_{1}+\omega_{2}\right) T_{0}\right) \\
& +E_{8}\left(T_{1}, T_{2}\right) \exp \left(i\left(2 \omega_{1}+\Omega\right) T_{0}\right)+E_{9}\left(T_{1}, T_{2}\right) \exp \left(i\left(2 \omega_{1}-\omega_{2}\right) T_{0}\right)+E_{10}\left(T_{1}, T_{2}\right) \\
& \times \exp \left(i\left(\Omega-2 \omega_{1}\right) T_{0}\right)+E_{11}\left(T_{1}, T_{2}\right) \exp \left(3 i \omega_{1} T_{0}\right)+E_{12}\left(T_{1}, T_{2}\right) \exp \left(i\left(3 \omega_{1}+\omega_{2}\right) T_{0}\right)+E_{13}\left(T_{1}, T_{2}\right) \\
& \times \exp \left(i\left(3 \omega_{1}+\Omega\right) T_{0}\right)+E_{14}\left(T_{1}, T_{2}\right) \exp \left(i\left(3 \omega_{1}-\omega_{2}\right) T_{0}\right)+E_{15}\left(T_{1}, T_{2}\right) \exp \left(i\left(-3 \omega_{1}+\Omega\right) T_{0}\right) \\
& +E_{16}\left(T_{1}, T_{2}\right) \exp \left(4 i \omega_{1} T_{0}\right)+E_{17}\left(T_{1}, T_{2}\right) \exp \left(i\left(4 \omega_{1}+\omega_{2}\right) T_{0}\right)+E_{18}\left(T_{1}, T_{2}\right) \exp \left(i\left(4 \omega_{1}+\Omega\right) T_{0}\right) \\
& +E_{19}\left(T_{1}, T_{2}\right) \exp \left(5 i \omega_{1} T_{0}\right)+E_{20}\left(T_{1}, T_{2}\right) \exp \left(i \Omega T_{0}\right)+E_{21}\left(T_{1}, T_{2}\right) \exp \left(i\left(\omega_{1}+\omega_{2}\right) T_{0}\right) \\
& +E_{22}\left(T_{1}, T_{2}\right) \exp \left(i\left(\Omega+\omega_{1}\right) T_{0}\right)+E_{23}\left(T_{1}, T_{2}\right) \exp \left(i\left(\omega_{1}-\omega_{2}\right) T_{0}\right)+E_{24}\left(T_{1}, T_{2}\right) \\
& \times \exp \left(i\left(\Omega-\omega_{1}\right) T_{0}\right)+E_{25}\left(T_{1}, T_{2}\right) \\
y_{2}= & E_{26}\left(T_{1}, T_{2}\right) \exp \left(2 i \omega_{1} T_{0}\right)+E_{27}\left(T_{1}, T_{2}\right) \exp \left(3 i \omega_{1} T_{0}\right)+E_{28}\left(T_{1}, T_{2}\right) \exp \left(4 i \omega_{1} T_{0}\right)+E_{29}\left(T_{1}, T_{2}\right) \\
& \times \exp \left(5 i \omega_{1} T_{0}\right)+E_{30}\left(T_{1}, T_{2}\right) \exp \left(i \Omega T_{0}\right) E_{31}\left(T_{1}, T_{2}\right) \exp \left(i \omega_{1} T_{0}\right)+E_{32}\left(T_{1}, T_{2}\right)
\end{aligned}
$$

where $E_{v}(v=1,2, \ldots, 32)$ are complex functions in $T_{1}$ and $T_{2}$, which are presented in the appendix (4). Then we can find the analytical solution of equations (1), (2) by substituting equations (13), (14), (17), (18), (19) and (20) into equations (3) and (4).

\subsection{Periodic Solution}

The simultaneous (primary and internal) resonance case $\left(\Omega \cong \omega_{1}, \omega_{2} \cong \omega_{1}\right)$ are investigated from the first-order approximation solution. By introducing small parameter as follow:

$$
\Omega=\omega_{1}+\sigma_{1}=\omega_{1}+\varepsilon \hat{\sigma}_{1} \text { and } \omega_{2}=\omega_{1}+\sigma_{2}=\omega_{1}+\varepsilon \hat{\sigma}_{2}
$$

Inserting Eq. (21) after using Eqs.(5)- (6) into the small-divisor and secular terms of Eqs. (15)-(16), we get the following solvability conditions:

$$
\begin{aligned}
& D_{1} A=-\frac{i \hat{F}}{4 \omega_{1}} e^{i \hat{\sigma}_{1} T_{1}}-\hat{\mu}_{1} A+\frac{3 \hat{\alpha}_{2} i}{2 \omega_{1}} \bar{A} A^{2}-\frac{\hat{\gamma} i}{2 \omega_{1}} B e^{i \hat{\sigma}_{2} T_{1}}+\frac{5 \hat{\alpha}_{4} i}{\omega_{1}} \bar{A}^{2} A^{3} \\
& D_{1} B=-\frac{i \hat{\lambda}}{2 \omega_{2}} A e^{-i \hat{\sigma}_{2} T_{1}}-\hat{\mu}_{2} B
\end{aligned}
$$

To investigate the solution of equations (22)- (23), it is suitable to refer the polar form of $A\left(T_{1}\right)$ and $B\left(T_{1}\right)$ as:

$$
A=\frac{a}{2} e^{i \beta_{1}}, B=\frac{b}{2} e^{i \beta_{2}}
$$

By inserting Eq. (24) into Eqs. (22)- (23), and after some mathematical manipulations, we get the autonomous equations of the amplitude and phase modulating: 
$\dot{a}=-\mu_{1} a+\frac{F}{2 \omega_{1}} \sin \left(\theta_{1}\right)+\frac{\gamma}{2 \omega_{1}} b \sin \left(\theta_{2}\right)$

$\dot{\theta}_{1}=\sigma_{1}-\frac{3 \alpha_{2}}{8 \omega_{1}} a^{2}+\frac{F}{2 \omega_{1} a} \cos \left(\theta_{1}\right)+\frac{\gamma}{2 \omega_{1} a} b \cos \left(\theta_{2}\right)-\frac{5 \alpha_{4}}{16 \omega_{1}} a^{4}$

$\dot{b}=-\mu_{2} b-\frac{\lambda}{2 \omega_{2}} a \sin \left(\theta_{2}\right)$

$\dot{\theta}_{2}=\sigma_{2}-\frac{3 \alpha_{2}}{8 \omega_{1}} a^{2}+\frac{F}{2 \omega_{1} a} \cos \left(\theta_{1}\right)+\frac{\gamma}{2 \omega_{1} a} b \cos \left(\theta_{2}\right)-\frac{5 \alpha_{4}}{16 \omega_{1}} a^{4}-\frac{\lambda}{2 \omega_{2} b} a \cos \left(\theta_{2}\right)$

where, $\theta_{1}=\hat{\sigma}_{1} T_{1}-\beta_{1}=\sigma_{1} t-\beta_{1}, \theta_{2}=\hat{\sigma}_{2} T_{1}-\beta_{1}+\beta_{2}=\sigma_{2} t-\beta_{1}+\beta_{2}$.

Thus $\dot{\theta}_{1}=\sigma_{1}-\dot{\beta}_{1}, \dot{\theta}_{2}=\sigma_{2}+\dot{\beta}_{2}-\dot{\beta}_{1}=\sigma_{2}+\dot{\beta}_{2}+\dot{\theta}_{1}-\sigma_{1}$.

\subsection{Equilibrium solution}

We have that at steady-state motion

$\dot{a}=\dot{\theta}_{1}=\dot{b}=\dot{\theta}_{2}=0$

Substituting Eqs. (29) into Eqs. (25) - (28), we get

$\mu_{1} a=+\frac{F}{2 \omega_{1}} \sin \left(\theta_{1}\right)+\frac{\gamma}{2 \omega_{1}} b \sin \left(\theta_{2}\right)$

$\sigma_{1} a=\frac{3 \alpha_{2}}{8 \omega_{1}} a^{3}-\frac{F}{2 \omega_{1}} \cos \left(\theta_{1}\right)-\frac{\gamma}{2 \omega_{1}} b \cos \left(\theta_{2}\right)+\frac{5 \alpha_{4}}{16 \omega_{1}} a^{5}$

$\mu_{2} b=-\frac{\lambda}{2 \omega_{2}} a \sin \left(\theta_{2}\right)$

$b\left(\sigma_{1}-\sigma_{2}\right)=-\frac{\lambda}{2 \omega_{2}} a \cos \left(\theta_{2}\right)$

By using Eqs. (30) - (33), we obtain

$$
\begin{aligned}
& b^{2}=\frac{\lambda^{2}}{4 \omega_{2}^{2}\left(\mu_{2}^{2}+\left(\sigma_{1}-\sigma_{2}\right)^{2}\right)} a^{2} \\
& \left(\mu_{1} a+Z_{1} a\right)^{2}+\left(a\left(\sigma_{1}-Z_{3}\right)-a^{3} Z_{2}-a^{5} Z_{4}\right)^{2}=\frac{F^{2}}{4 \omega_{1}^{2}}
\end{aligned}
$$

Where

$$
Z_{1}=\frac{\gamma \mu_{2} \lambda}{4 \omega_{1} \omega_{2}\left(\mu_{2}^{2}+\left(\sigma_{1}-\sigma_{2}\right)^{2}\right)}, Z_{2}=\frac{3 \alpha_{2}}{8 \omega_{1}}, Z_{3}=\frac{\gamma\left(\sigma_{1}-\sigma_{2}\right) \lambda}{4 \omega_{1} \omega_{2}\left(\mu_{2}^{2}+\left(\sigma_{1}-\sigma_{2}\right)^{2}\right)}, Z_{4}=\frac{5 \alpha_{4}}{16 \omega_{1}} \text {. }
$$

Equations (34) and (35) are the FRE that utilized to characterize the steady state solutions conductance for the practical state i.e. $(a \neq 0, b \neq 0)$. 


\subsection{Stability analysis}

To define the stability of the steady-state solution, present the following forms: $a=a_{1}+a_{0}, b=b_{1}+b_{0}, \theta_{1}=\theta_{11}+\theta_{10}, \theta_{2}=\theta_{21}+\theta_{20}$

Substituting from Eq. (36) into Eqs. (25) - (28), and consideration only the linear terms in $a_{1}, \theta_{11}, b_{1}$ and $\theta_{21}$, we get the next equations that can be established in the matrix form as:

$\left[\begin{array}{l}\dot{a}_{1} \\ \dot{\theta}_{11} \\ \dot{b_{1}} \\ \dot{\theta}_{21}\end{array}\right]=\left[\begin{array}{llll}r_{11} & r_{12} & r_{13} & r_{14} \\ r_{21} & r_{22} & r_{23} & r_{24} \\ r_{31} & r_{32} & r_{33} & r_{34} \\ r_{41} & r_{42} & r_{43} & r_{44}\end{array}\right]\left[\begin{array}{l}a_{1} \\ \theta_{11} \\ b_{1} \\ \theta_{21}\end{array}\right]$

where the above square matrix is the Jacobian matrix, $r_{l m}, l=1,2,3,4$ and $m=1,2,3,4$ are given in the Appendix. The eigenvalues of the above system can be illustrated as:

$\lambda^{4}+r_{1} \lambda^{3}+r_{2} \lambda^{2}+r_{3} \lambda+r_{4}=0$

\section{The method of phase portrait for the system.}

In this section we discuss the phase plan form that can be characterized by two equations of nonlinear first order for the main system without control i.e $b=0, \theta_{2}=0$ as follows:

$$
\begin{aligned}
& \dot{a}=f_{1}\left(a, \theta_{1}\right)=-\mu_{1} a+\frac{F}{2 \omega_{1}} \sin \left(\theta_{1}\right)=0 \\
& \dot{\theta}_{1}=f_{2}\left(a, \theta_{1}\right)=\sigma_{1} a-\frac{3 \alpha_{2}}{8 \omega_{1}} a^{3}-\frac{5 \alpha_{4}}{16 \omega_{1}} a^{5}+\frac{F}{2 \omega_{1}} \cos \left(\theta_{1}\right)=0
\end{aligned}
$$

On the Poincare phase plane, the phase orbits, which are represented by the vector fields corresponding to equations (39) and (40), provide useful data about the properties of the main system. We can put the equations (39) and (40) in the form $\dot{V}=J V$, where $J$ is the Jacobian matrix as follows:

$V=\left[\begin{array}{c}a \\ \theta_{1}\end{array}\right], \dot{V}=\left[\begin{array}{c}\dot{a} \\ \dot{\theta}_{1}\end{array}\right], J=\left[\begin{array}{ll}\frac{\partial f_{1}}{\partial a}=-\mu_{1} & \frac{\partial f_{1}}{\partial \theta_{1}}=+\frac{F}{2 \omega_{1}} \cos \left(\theta_{1}\right) \\ \frac{\partial f_{2}}{\partial a}=\sigma_{1}-\frac{9 \alpha_{2}}{8 \omega_{1}} a^{2}-\frac{25 \alpha_{4}}{16 \omega_{1}} a^{4} & \frac{\partial f_{2}}{\partial \theta_{1}}=-\frac{F}{2 \omega_{1}} \sin \left(\theta_{1}\right)\end{array}\right]$

We can look for equilibrium points, and define whether the function changes toward or away from these points as time passes giving rise to the asymptotic behavior of the function without having to solve for it.

If we put $\dot{a}=0, \dot{\theta}_{1}=0$ in system (39) and (40) solving it, we get the critical (equilibrium or rest) points at the values of system parameters as $\mu_{1}=0.05, \alpha_{2}=1, \alpha_{4}=0.05$, $F=0.5, \omega_{1}=5, \sigma_{1}=0$ that are $(0.753646,0.853591+6.28319 \mathrm{k}), \mathrm{k} \in$ integer. By using these critical points we will explain the phase portrait classifications for values of the eigenvalues $\lambda_{1}, \lambda_{2}$ which obtained from $\operatorname{det}[J-\lambda I]=0$. Because of the eigenvalues take the complex formula $\left(\lambda_{1,2}=c \pm i d, c<0, d>0\right)$, the equilibrium point is classified as the asymptotically stable spiral (spirals in) point at $(0.753646,0.853591+6.28319 \mathrm{k}),(\mathrm{k} \in$ Integers) that illustrated in figure 2 as in Ref [21]. If we change the values of system parameters this will lead to change the critical points and there eigenvalues classifications that can be summarized as in table 1 . 
Table [1]: Equilibria classification for almost linear systems

\begin{tabular}{|c|c|}
\hline Eigenvalues of $\mathbf{J}$ & Nonlinear classification \\
\hline$\lambda_{1}<0<\lambda_{2}$ & Unstable saddle \\
\hline$\lambda_{1}<\lambda_{2}<0$ & Stable proper node \\
\hline$\lambda_{1}>\lambda_{2}>0$ & Unstable proper node \\
\hline$\lambda_{1}=\lambda_{2}<0$ & Stable improper node or spiral \\
\hline$\lambda_{1}=\lambda_{2}>0$ & Unstable improper node or spiral \\
\hline$\lambda_{1}=\bar{\lambda}_{2}=c+i d, c<0, d>0$ & Stable spiral \\
\hline$\lambda_{1}=\lambda_{2}=c+i d, c>0, d>0$ & Unstable spiral \\
\hline$\lambda_{1}=\bar{\lambda}_{2}=i d, d>0$ & Stable or unstable ,center or spiral \\
\hline
\end{tabular}
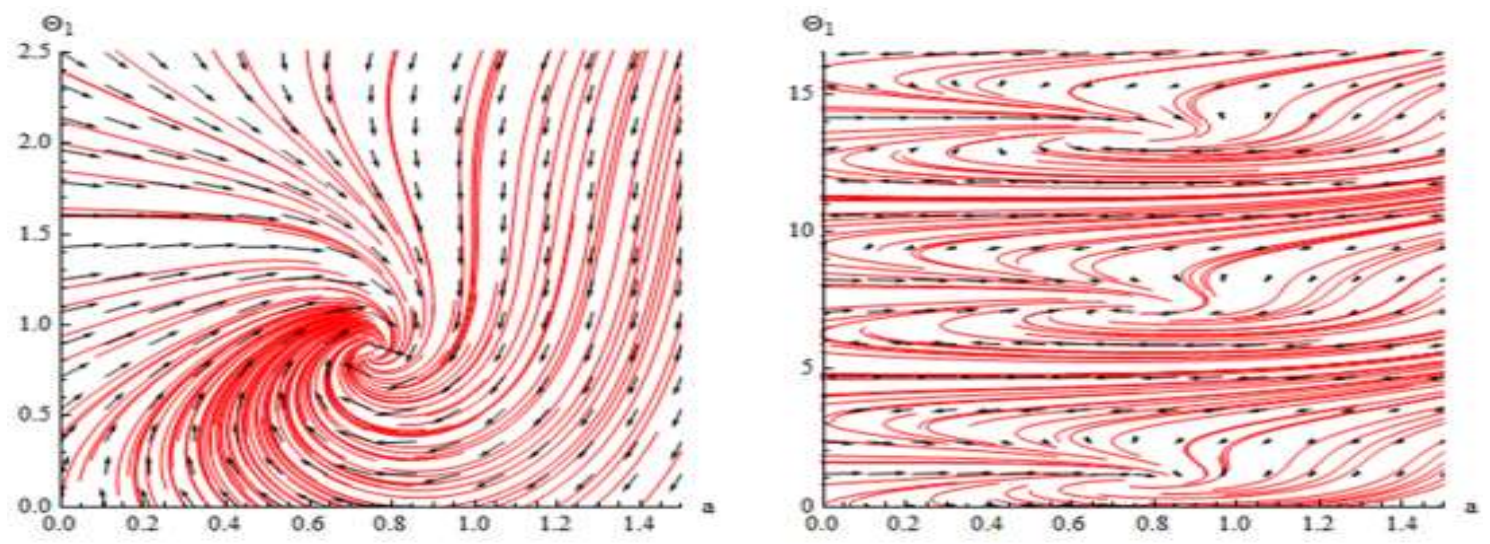

Fig.2 phase plane of the uncontrolled system

\section{RESULTS AND DISCUSSIONS}

\subsection{Time History}

Based on MATLAB computer program in this section, we simulated numerically equations (1)(2) which introduced the nonlinear dynamical model without and with involved PPF control to show the reduce of vibration after adding this control. After inserting the values of parameters as $\mu_{1}=0.05, \alpha_{1}=0.5, \alpha_{2}=1, \alpha_{3}=1, \alpha_{4}=0.05, F=0.5, \Omega=\omega_{1}, \mu_{2}=0.005, \lambda=5, \gamma=10$, $\omega_{1}=5, \omega_{2}=\omega_{1}$ the time history can be illustrated as in Figs. (3), (4). Fig. (3) represents the uncontrolled amplitude time history and phase plane at primary resonance of the main model. Fig. (4) illustrates the time histories of both controlled amplitude of the main model and PPF amplitude also, phase plane of both the main system and controller at primary and 1:1 internal resonance. We notice that from Fig. (2) after using the PPF controller the oscillations amplitude for the basic model reaches to 0.0005091 , this interpret that the oscillations suppressed to about $99.93 \%$ from its value without PPF control after short time $t=150 \mathrm{Sec}$. This leads to the effectiveness of the absorber $E_{a}\left(E_{a}=\right.$ the system steady-state amplitude before controller / the system steady-state amplitude after controller) is around 1478.099 after using PPF controller for the essential system. 

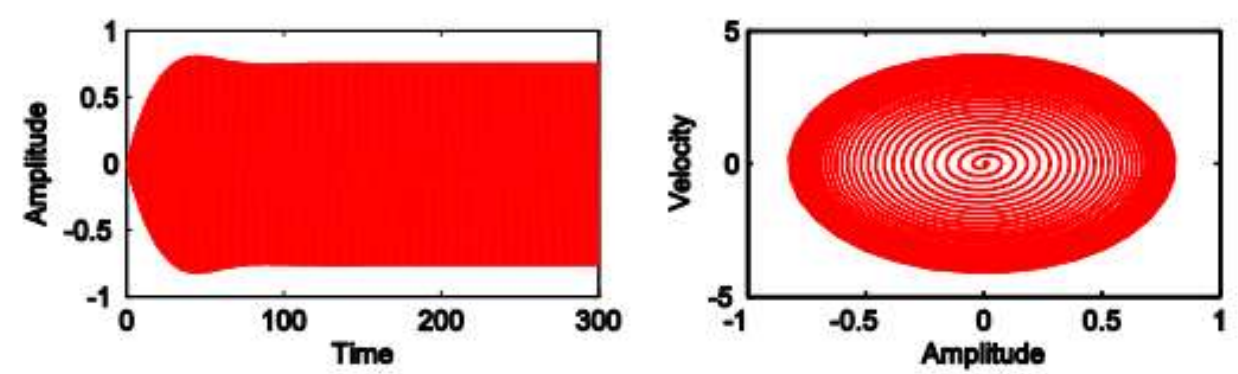

Fig.3 Time History of Uncontrolled system vibration amplitude at primary resonance
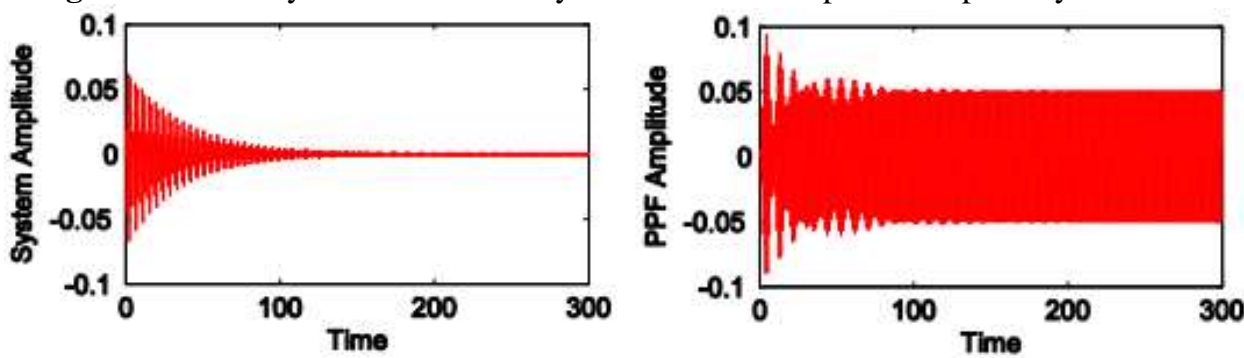

Fig.4 The main system with PPF control time History at primary and 1:1 internal resonance

\subsection{Comparison of time history between numerical and analytical solutions}

Applying the condition for a steady-state solution, that is, $\dot{a}=\dot{b}=\dot{\theta}_{1}=\dot{\theta}_{2}=0$ at the $1: 1$ internal and primary resonance, the comparison between the numerical solutions of equations (1) and (2) for PPF control and the analytical solutions given by equations (31) - (34) has been declared as in Fig.5. The dashed lines indicate the amplitudes modulation $a, b$ for the generalized coordinate $x, y$. On the other hand, the continuous lines characterize the time history of oscillations, which acquired numerically as solutions of the equations of the system with PPF controller. From this figure, we found that the two studying cases of resonance are applicable to each other at the steady state solution. Also, there is a good agreement between approximate and numerical solutions.
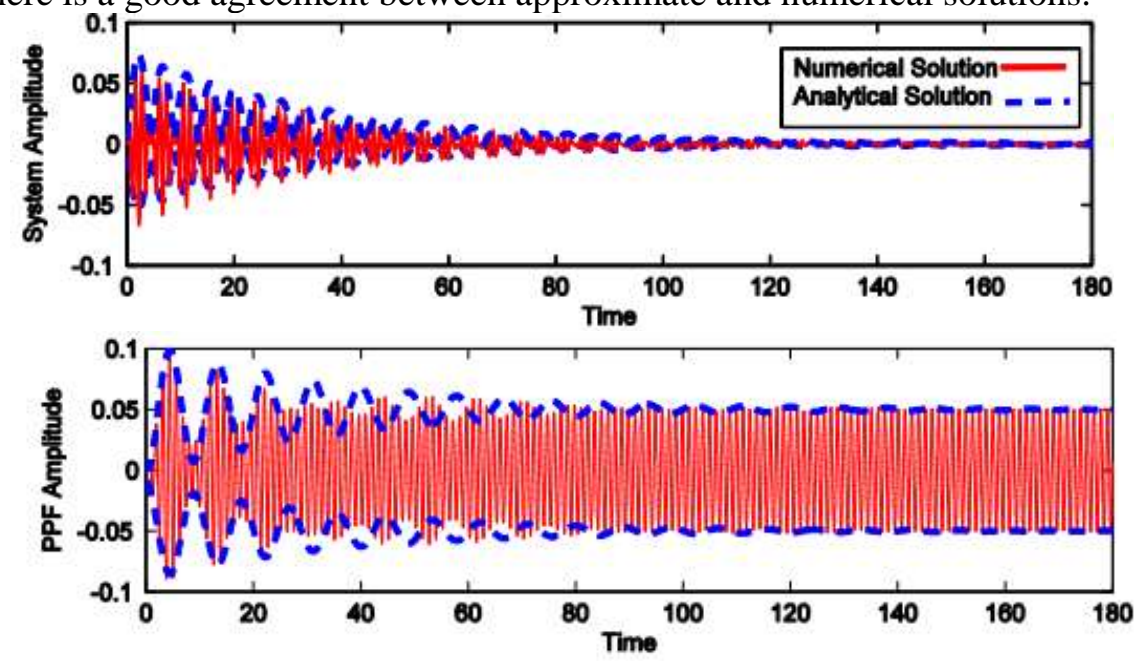

Fig.5 Comparison between numerical simulation and perturbation analysis for the main system with PPF control

\subsection{Effects of various parameters on the system steady-state behavior}

Fig.6 shows that the FRC for the controlled system at the practical case $(a \neq 0, b \neq 0)$, where the dashed line is an unstable area and the continuous line is a stable region. Also, we observed that 
the FRC contain two beaks lies on left and right $\sigma_{1}=0$.Fig. 6a displays the steady-state amplitude for the essential model ( $a$ against $\sigma_{1}$ ) and Fig. 6b displays the steady-state amplitude for PPF controller ( $b$ against $\sigma_{1}$ ). At increasing the values of the damping coefficient $\mu_{1}$ as illustrated in Figs. $7 \mathrm{a}$ and $7 \mathrm{~b}$, the beaks of steady state amplitudes of the essential system and controller respectively decreases. The effects of the linear natural frequency $\omega_{1}$ for essential model and controller appears in Figs.8a and 8b. By decreasing the numbers of the linear natural frequency, the beaks of essential system and controller amplitudes increase, the unstable region increases and the bandwidth of vibration suppression for the essential system and the controller increases. Fig.9 and Fig.10 shows that when changing the sign of non-linear coefficient $\alpha_{2}$ and $\alpha_{4}$ respectively, the amplitude for the essential system $a$ and the amplitude of absorber $b$ is curved to the right with positive sign denoting a hardening-type spring nonlinearity. On the other wise, with negative sign denoting a softing-type spring nonlinearity occurred when the amplitude for the essential system $a$ and the amplitude of absorber $b$ is curved to the left. In Fig. 11(a,b), we display the FRC of the harmonic force amplitude $F$ for the essential system and the restrainer, respectively. Also, this figure shows that the more increasing in the harmonic force amplitude the more bending away of the FRC away from the linear curves and the more increasing the beaks of amplitudes. The unstable regions are increased with increasing the external force.

Fig. $12(\mathrm{a}, \mathrm{b})$ indicates that for growing values of the damping factor for the absorber $\mu_{2}$, the steady-state amplitudes for the essential model and the absorber are reduced, and the unstable region is decreased until total figure become stable. Also, we noticed that at $\sigma_{1}=0$ the amplitude of the main system moving away from zero, which is supposed to reach zero at primary resonance case $\Omega=\omega_{1}$ that occurs at $\sigma_{1}=0$, therefore, it is preferable to take a small value for the variable $\mu_{2}$. The bandwidths are gradually appearing for increasing the control signal gain $\gamma$ and the feedback signal gain $\lambda$ values as illustrated in Fig. 13 and Fig.14, respectively. Also, the peaks amplitudes of PPF control are reducing at increasing the number of $\gamma$ as in Fig. 13b. on the other hand, the peaks amplitudes of PPF control are increasing at growing the value of $\gamma$ as in Fig. 14b.

At the detuning parameter $\sigma_{2}$ is increased as in Figs. 15a and 15b, the amplitudes of the essential model and PPF moved to right and the amplitude peak value of right-hand control and the amplitude peak value of left-hand side are growing and reducing. Using the curves of frequency response, we concluded that the value of $\sigma_{2}$ must be equal the value of $\sigma_{1}$ to obtain the smallest value of the steady-state amplitude of essential system and the absorber which cleared at $\sigma_{2}=\sigma_{1}=-1$, $\sigma_{2}=\sigma_{1}=0$ and $\sigma_{2}=\sigma_{1}=1$.In Fig. 16 we using MATLAB ${ }^{\circledR}$ computer program to appear the rapprochement between the FRC and the numerical solution by the small circles. From this figure, all predictions based on evidence of the analytical solution are at extremely valid coincidence with the numerical solution.

In Figure 17, a comparison is made between FRC before and after adding control. Note that after the addition of control, the amplitude of the system decreases at the measured resonance i.e when $\sigma_{1}=0$, and two beaks are shown on the sides of the $\sigma_{1}=0$. 

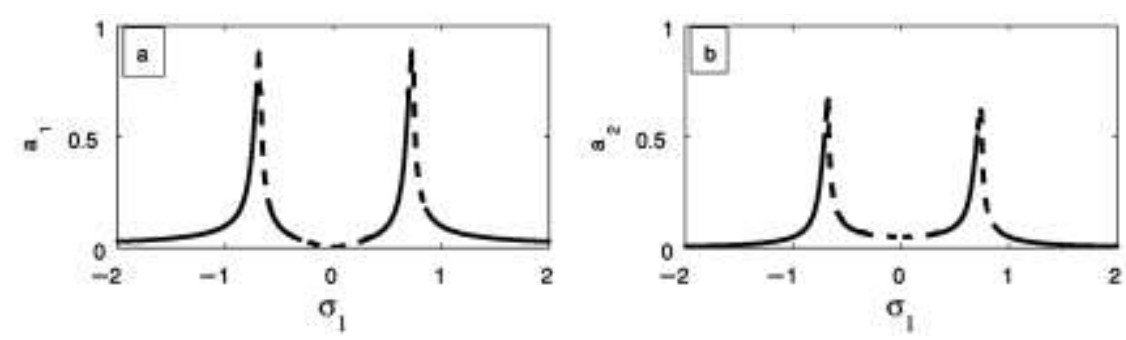

Fig.6 The FRC of Controlled system (a) ( $a$ against $\sigma_{1}$ ), and (b) ( $b$ against $\sigma_{1}$ )
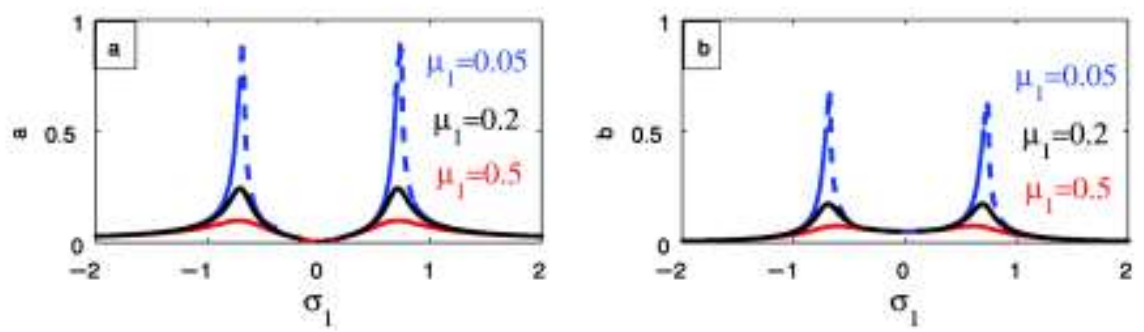

Fig.7. Damping coefficient $\mu_{1}$ effects: (a) the main system amplitude $a$ (b) PPF amplitude $b$.
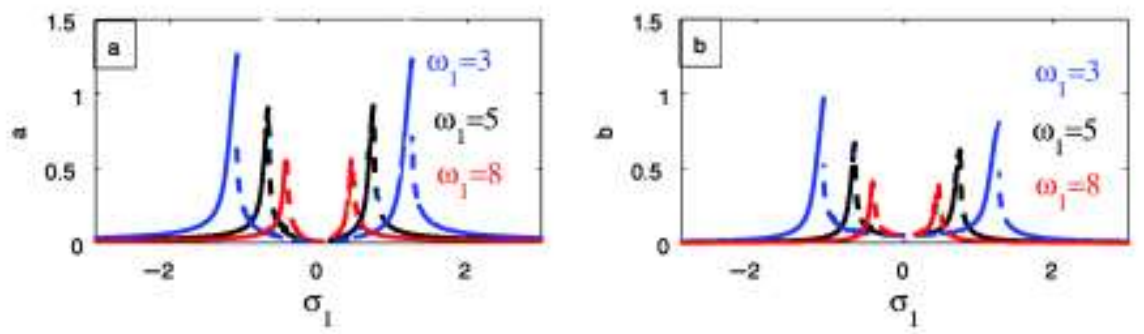

Fig.8. Natural frequency $\omega_{1}$ effects: (a) the main system amplitude $a$ (b) PPF amplitude $b$.
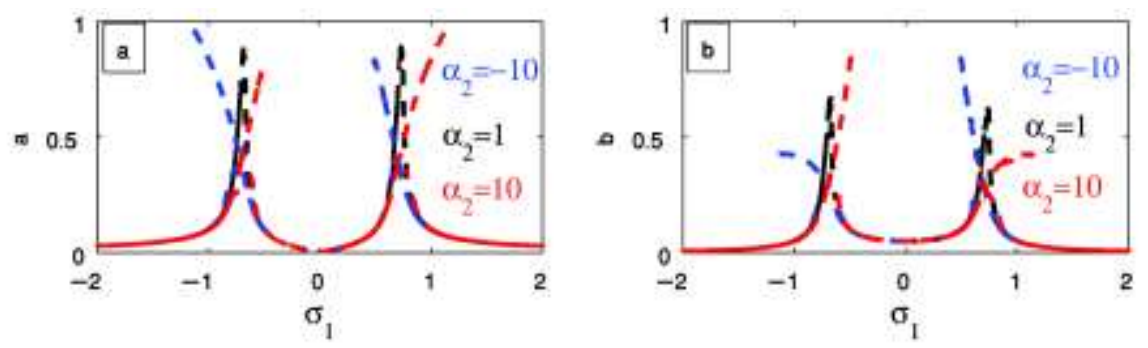

Fig.9. Nonlinear coefficient $\alpha_{2}$ effects: (a) the main system amplitude $a$ (b) PPF amplitude $b$.
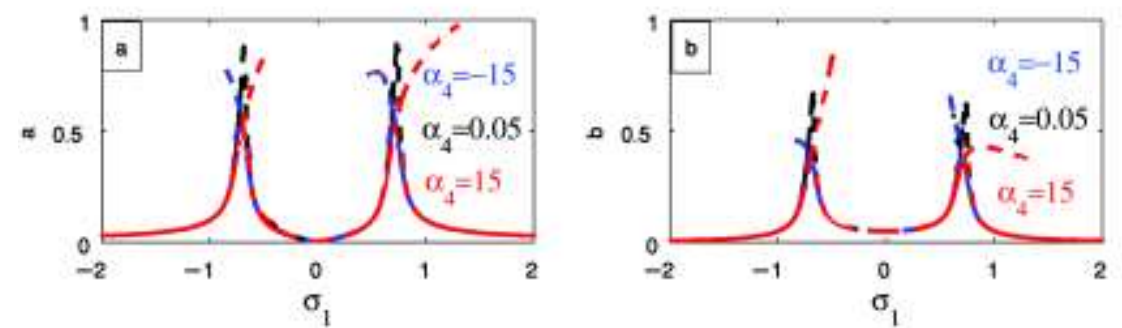

Fig.10. Nonlinear coefficient $\alpha_{4}$ effects: (a) the main system amplitude $a$ (b) PPF amplitude $b$. 

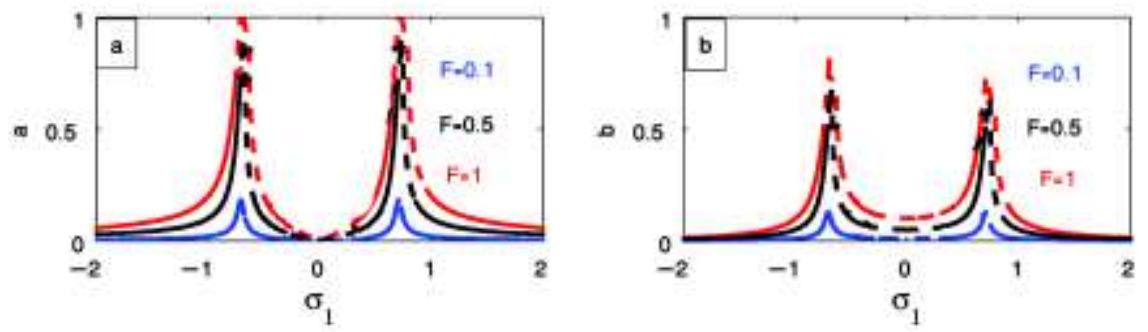

Fig.11. External force $F_{1}$ effects: (a) the main system amplitude $a$ (b) PPF amplitude $b$.
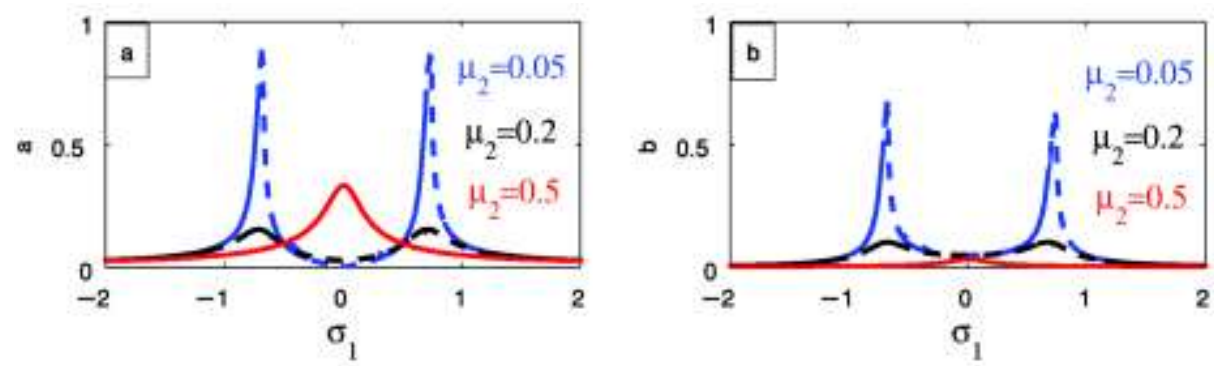

Fig.12. Damping coefficient $\mu_{2}$ effects: (a) the main system amplitude $a$ (b) PPF amplitude $b$.
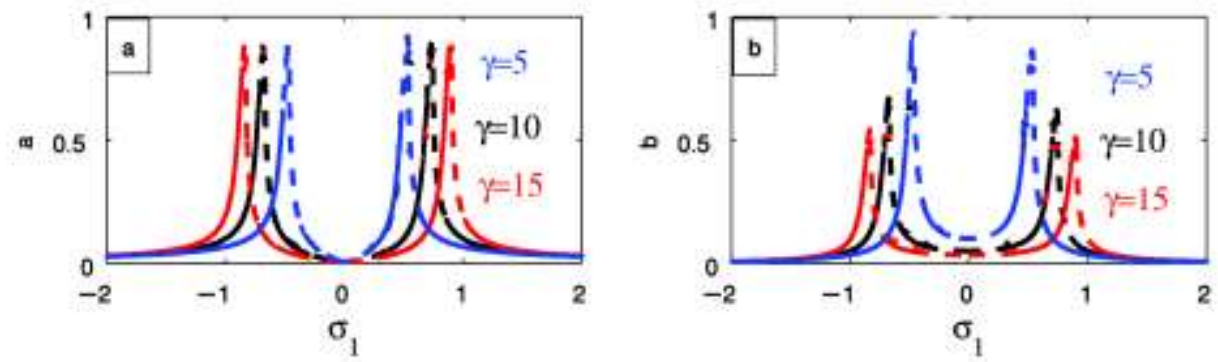

Fig.13. Control signal gains $\gamma$ effects: (a) the main system amplitude $a$ (b) PPF amplitude $b$.
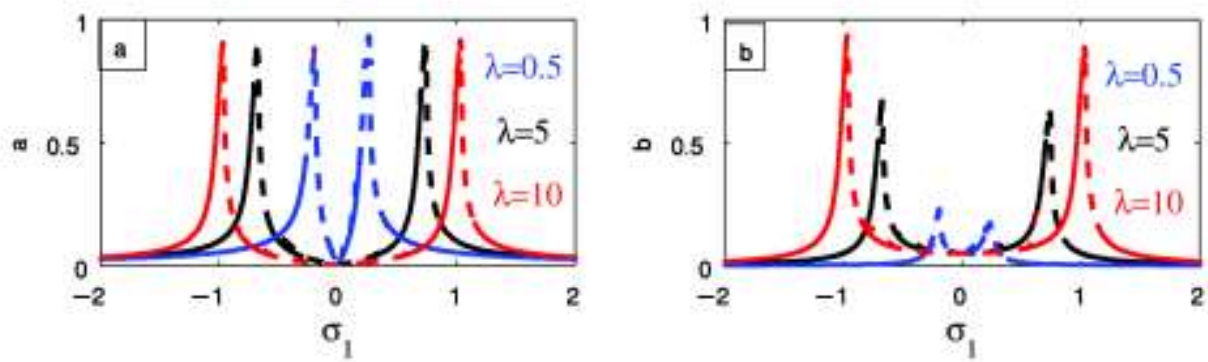

Fig.14. Feedback signal gains $\lambda$ effects: (a) the main system amplitude $a$ (b) PPF amplitude $b$
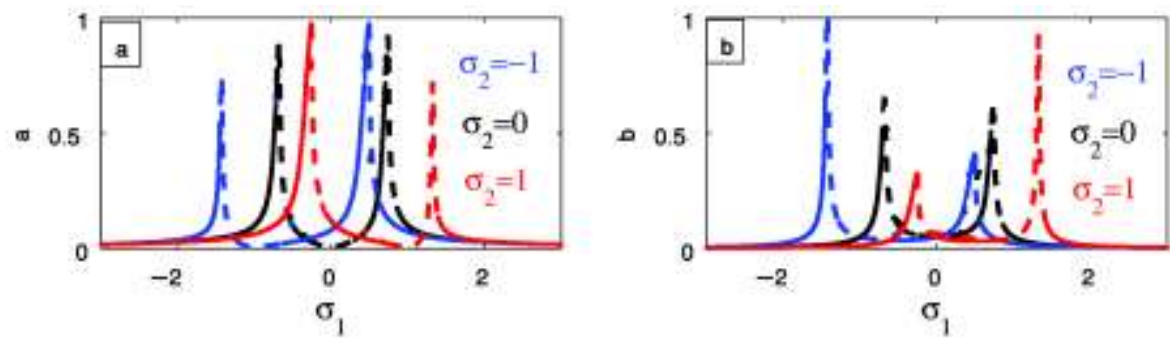

Fig.15. Detuning parameter $\sigma_{2}$ effects: (a) the main system amplitude $a$ (b) PPF amplitude $b$. 

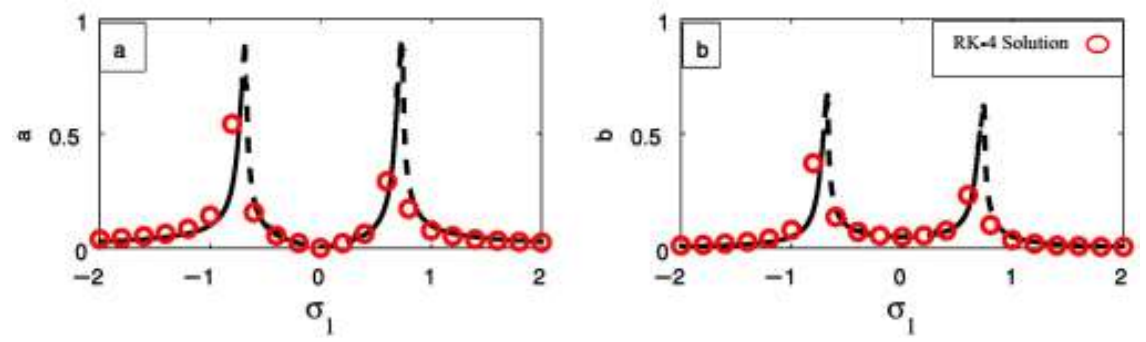

Fig.16. FRC and numerical solutions (a) ( $a$ against $\left.\sigma_{1}\right)$, and (b) $\left(b\right.$ against $\left.\sigma_{1}\right)$

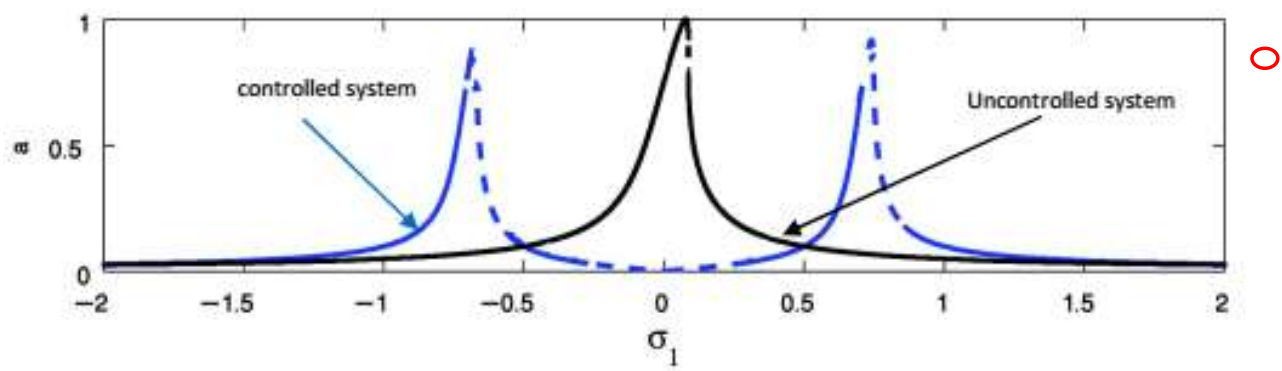

Fig.17. Comparison between the FRC of an Uncontrolled system and controlled system

\section{Comparison between the previous works and this work}

a. In a previous work [7], the authors investigated a non-linear s.d.o.f. model under an external excitation with two distinct time-delays to analysis of superharmonic, primary of order five, and sub harmonic of order one three resonances. In each studied resonance, the solutions of steadystate and their stabilities are determined. They discussed the effect of time-delays and the feedback gains of the system and they enhanced the perfect appropriate feedback..

b. Firstly, we are adding PPF control equation to the non-linear s.d.o.f. equation an external excitation mentioned in Ref. [7] to control the vibration model as shown in this work.

c. Secondly, we applied the MSPT to get a solution of the studied system and examined the stability of this system.

d. Finally, we have succeeded in decreasing the amplitude of steady-state for the main system to 99.93\% after using PPF absorber from its value before absorber. For obtaining the effective PPF controller, we have found that it is essential tuning the external excitation frequency to the controller natural frequency ( $\Omega=\omega_{2}$ ).

\section{CONCLUSION}

The nonlinear dynamical system connected to PPF was introduced with two coupled differential equations. These equations have been solved analytically by using MSPT approximation. The FRE has been obtained near the primary resonance and 1:1 iinternal resonance. The time development of the amplitudes and phases of both the essential model and the absorber are represented by four first order differential equations that derived by MSPT. After that we have investigated the influences of the parameters to present the amplitude performance of the model and PPF. The stability study is completed to define the stable boundary of the control variables. From this research, the highlighted points can be summarized as following:

1. By using PPF controller, the amplitude of steady-state is decreased to $99.93 \%$ from its value before control.

2. The effectiveness of the absorber $E_{a}$ is about 1478.099 when using PPF controller for the main system.

3. The amplitudes of the essential system $a$ and the controller $b$ are increased when increasing the values of $F$. 
4. The FRC of the model and the absorber are curved to the right denoting a hardening-type spring nonlinearity when changing the sign to positive value of $\alpha_{2}$ and $\alpha_{4}$. On the other wise, with negative sign of $\alpha_{2}$ and $\alpha_{4}$ the FRC of the system and the controller are curved to the left denoting a softening-type spring nonlinearity.

5. The best performance for the amplitude vibration reduction which reach to zero when $\sigma_{2}=\sigma_{1}$ ( $\left.\Omega=\omega_{1}\right)$.

There are good agreements when make a comparison between the approximate and the numerical solutions at time history and the FRC as presented in Figs. 3 and 14 at the resonance case of study, respectively.

\section{Appendix}

$$
\begin{aligned}
& C_{1}=\frac{\hat{\gamma} B}{\omega_{1}^{2}-\omega_{2}^{2}}, C_{2}=\frac{\left(-\hat{\alpha}_{1} A^{2}-4 \hat{\alpha}_{3} A^{3} \bar{A}\right)}{5 \omega_{1}^{2}}, C_{3}=\frac{\left(-\hat{\alpha}_{2} A^{3}-5 \hat{\alpha}_{4} A^{4} \bar{A}\right)}{8 \omega_{1}^{2}}, C_{4}=\frac{-\hat{\alpha}_{3} A^{4}}{15 \omega_{1}^{2}} \\
& , C_{5}=\frac{-\hat{\alpha}_{4} A^{5}}{15 \omega_{1}^{2}}, C_{6}=\frac{\left(-\hat{\alpha}_{1} A \bar{A}-3 \hat{\alpha}_{3} A^{2} \bar{A}^{2}\right)}{\omega_{1}^{2}}, C_{7}=\frac{\hat{F}}{2\left(\omega_{1}^{2}-\Omega^{2}\right)}, C_{8}=\frac{\hat{\lambda} A}{\omega_{2}^{2}-\omega_{1}^{2}} \\
& E_{1}=\frac{5 \hat{\alpha}_{4} A^{4} C_{5}}{80 \omega_{1}^{2}}, E_{2}=\frac{1}{-63 \omega_{1}^{2}}\left(-A^{3}\left(4 \hat{\alpha}_{3} C_{5}+5 A \hat{\alpha}_{4} C_{4}\right)\right) \text {, } \\
& E_{3}=\frac{1}{-35 \omega_{1}^{2}}\left(-A\left(2 C_{5}\left(\hat{\alpha}_{1}+6 A \bar{A} \hat{\alpha}_{3}\right)+A\left(A\left(4 C_{3} \hat{\alpha}_{3}+5 A C_{2} \hat{\alpha}_{4}\right)+C_{4}\left(3 \hat{\alpha}_{2}+20 A \bar{A} \hat{\alpha}_{4}\right)\right)\right)\right), \\
& E_{4}=\frac{1}{-48 \omega_{1}^{2}}\left(-A^{2}\left(A\left(4 C_{4} \hat{\alpha}_{3}+5 A C_{3} \hat{\alpha}_{4}\right)+C_{5}\left(3 \hat{\alpha}_{2}+20 A \bar{A} \hat{\alpha}_{4}\right)\right)\right) \text {, } \\
& E_{5}=\frac{1}{\omega_{1}^{2}-\omega_{2}^{2}}\left(-2\left(3 A C_{1} \bar{A} \hat{\alpha}_{2}+15 A^{2} \bar{A}^{2} C_{1} \hat{\alpha}_{4}+i \omega_{2}\left(C_{1} \mu_{1}+D_{1} C_{1}\right)\right)\right), \\
& E_{6}=\frac{1}{-3 \omega_{1}^{2}}\left(-3 \bar{A}^{2} C_{4} \hat{\alpha}_{2}-4 \bar{A}^{3} C_{5} \hat{\alpha}_{3}-2 \bar{A} C_{3}\left(\hat{\alpha}_{1}+6 A \bar{A} \hat{\alpha}_{3}\right)-5 A^{4} \bar{C}_{2} \hat{\alpha}_{4}-20 A^{3} \bar{A} \bar{C}_{7} \hat{\alpha}_{4}\right. \\
& \left.-3 A^{2}\left(\bar{C}_{7} \hat{\alpha}_{2}+10 \bar{A}^{2} C_{2} \hat{\alpha}_{4}\right)-2 A\left(3 \bar{A} C_{2} \hat{\alpha}_{2}+10 \bar{A}^{3} C_{4} \hat{\alpha}_{4}\right)-4 i C_{2} \hat{\mu}_{1} \omega_{1}-4 i D_{1} C_{2} \omega_{1}\right), \\
& E_{7}=\frac{1}{-3 \omega_{1}^{2}-\omega_{2}\left(\omega_{2}+4 \omega_{1}\right)}\left(-A^{2} C_{1}\left(3 \hat{\alpha}_{2}+20 \bar{A} A \hat{\alpha}_{4}\right)\right) \\
& E_{8}=\frac{1}{-3 \omega_{1}^{2}-\Omega\left(\Omega+4 \omega_{1}\right)}\left(-A^{2} C_{6} C_{7}\left(3 \hat{\alpha}_{2}+20 \bar{A} A \hat{\alpha}_{4}\right)\right) \text {, } \\
& E_{9}=\frac{1}{-3 \omega_{1}^{2}+\omega_{2}\left(\omega_{2}-4 \omega_{1}\right)}\left(-A^{2} \bar{C}_{1}\left(3 \hat{\alpha}_{2}+20 \bar{A} A \hat{\alpha}_{4}\right)\right) \\
& E_{10}=\frac{1}{-3 \omega_{1}^{2}-\Omega\left(\Omega-4 \omega_{1}\right)}\left(-\bar{A}^{2} C_{6}\left(3 \hat{\alpha}_{2}+20 \bar{A} A \hat{\alpha}_{4}\right)\right), \\
& E_{11}=\frac{1}{-8 \omega_{1}^{2}}\left(-2 \bar{A} C_{4} \hat{\alpha}_{1}-3 \bar{A}^{2} C_{5} \hat{\alpha}_{2}-4 A^{3}\left(C_{7}+\bar{C}_{7}\right) \hat{\alpha}_{3}-6 A^{2} \bar{A}\left(2 C_{2} \hat{\alpha}_{3}+5 C_{3} \bar{A} \hat{\alpha}_{4}\right)-2 A\left(C_{2} \hat{\alpha}_{1}\right.\right.
\end{aligned}
$$


$\left.\left.+\bar{A}\left(3 C_{3} \hat{\alpha}_{2}+6 C_{4} \bar{A} \hat{\alpha}_{3}+10 C_{5} \bar{A}^{2} \hat{\alpha}_{4}\right)\right)-6 i \omega_{1} \hat{\mu}_{1} C_{3}-6 i \omega_{1} D_{1} C_{3}\right)$,

$$
E_{12}=\frac{4 A^{3} C_{1} \hat{\alpha}_{3}}{8 \omega_{1}^{2}+\omega_{2}\left(\omega_{2}+6 \omega_{1}\right)}, E_{13}=\frac{4 A^{3} C_{6} \hat{\alpha}_{3}}{8 \omega_{1}^{2}+\Omega\left(\Omega-6 \omega_{1}\right)},
$$

$$
E_{14}=\frac{4 A^{3} \bar{C}_{1} \hat{\alpha}_{3}}{8 \omega_{1}^{2}+\omega_{2}\left(\omega_{2}-6 \omega_{1}\right)}, E_{15}=\frac{4 \bar{A}^{3} C_{6} \hat{\alpha}_{3}}{8 \omega_{1}^{2}+\Omega\left(\Omega-6 \omega_{1}\right)},
$$

$$
\begin{aligned}
E_{16} & =\frac{1}{-15 \omega_{1}^{2}}\left(-2 A C_{3} \hat{\alpha}_{1}-2 \bar{A} C_{5} \hat{\alpha}_{1}-3 A^{2} C_{2} \hat{\alpha}_{2}-6 A \bar{A} C_{4} \hat{\alpha}_{2}-12 A^{2} \bar{A} C_{3} \hat{\alpha}_{3}-5 A^{4} C_{7} \hat{\alpha}_{4}\right. \\
& \left.-12 A \bar{A}^{2} C_{5} \hat{\alpha}_{3}-20 A^{3} \bar{A} C_{2} \hat{\alpha}_{4}-30 A^{2} \bar{A}^{2} C_{4} \hat{\alpha}_{4}-5 A^{4} \bar{C}_{7} \hat{\alpha}_{4}-8 i \hat{\mu}_{1} \omega_{1} C_{4}-8 i \omega_{1} D_{1} C_{4}\right),
\end{aligned}
$$

$$
E_{17}=\frac{5 A^{4} C_{1} \hat{\alpha}_{4}}{15 \omega_{1}^{2}+\omega_{2}\left(\omega_{2}+8 \omega_{1}\right)}, E_{18}=\frac{5 \bar{A}^{4} C_{6} \hat{\alpha}_{4}}{15 \omega_{1}^{2}+\Omega\left(\Omega-8 \omega_{1}\right)},
$$

$$
E_{19}=\frac{1}{-24 \omega_{1}^{2}}\left(-2 A\left(C_{4} \hat{\alpha}_{1}+3 C_{5} \bar{A} \hat{\alpha}_{2}\right)-4 A^{3}\left(C_{2} \hat{\alpha}_{3}+5 C_{3} \bar{A} \hat{\alpha}_{4}\right)\right.
$$

$$
\left.-3 A^{2}\left(C_{3} \hat{\alpha}_{2}+2 \bar{A}\left(2 C_{4} \hat{\alpha}_{3}+5 C_{5} \bar{A} \hat{\alpha}_{4}\right)\right)-10 i \omega_{1}\left(C_{5} \hat{\mu}_{1}+D_{1} C_{5}\right)\right),
$$

$$
E_{20}=\frac{1}{\omega_{1}^{2}-\Omega^{2}}\left(-2\left(3 A C_{6} \bar{A} \hat{\alpha}_{2}+15 A^{2} \bar{A}^{2} C_{6} \hat{\alpha}_{4}+i \Omega\left(C_{6} \mu_{1}+D_{1} C_{6}\right)\right)\right) \text {, }
$$$$
E_{21}=\frac{1}{-\omega_{2}\left(\omega_{2}+2 \omega_{1}\right)}\left(-2 A C_{1} \hat{\alpha}_{1}-12 A^{2} C_{1} \bar{A} \hat{\alpha}_{3}\right),
$$$$
E_{22}=\frac{1}{-\Omega\left(\Omega+2 \omega_{1}\right)}\left(-2 A C_{6} \hat{\alpha}_{1}-12 A^{2} C_{6} \bar{A} \hat{\alpha}_{3}\right),
$$

$$
E_{23}=\frac{1}{-\omega_{2}\left(\omega_{2}-2 \omega_{1}\right)}\left(-2 A \bar{C}_{1}\left(\hat{\alpha}_{1}+6 A \bar{A} \hat{\alpha}_{3}\right)\right),
$$

$$
E_{24}=\frac{1}{-\Omega\left(\Omega-2 \omega_{1}\right)}\left(-2 \bar{A} C_{6}\left(\hat{\alpha}_{1}+6 A \bar{A} \hat{\alpha}_{3}\right)\right),
$$$$
E_{25}=\frac{1}{\omega_{2}^{2}}\left(-6 \hat{\alpha}_{2} A C_{7} \bar{A}-3 \hat{\alpha}_{2} A^{2} \bar{C}_{2} \bar{A}-4 \hat{\alpha}_{3} A^{3} \bar{C}_{3}-30 \hat{\alpha}_{4} A^{2} \bar{A}^{2} C_{7}\right.
$$$$
\left.-20 \hat{\alpha}_{4} A \bar{A}^{3} C_{2}-5 \hat{\alpha}_{4} A^{4} \bar{C}_{4}\right),
$$

$E_{26}=\frac{\hat{\lambda} C_{2}}{\omega_{2}^{2}-4 \omega_{1}^{2}}, E_{27}=\frac{\hat{\lambda} C_{3}}{\omega_{2}^{2}-9 \omega_{1}^{2}}, E_{28}=\frac{\hat{\lambda} C_{4}}{\omega_{2}^{2}-16 \omega_{1}^{2}}, E_{29}=\frac{\hat{\lambda} C_{5}}{\omega_{2}^{2}-25 \omega_{1}^{2}}$,

$$
E_{30}=\frac{\hat{\lambda} C_{6}}{\omega_{2}^{2}-\Omega^{2}}, E_{31}=\frac{\left(-2 i C_{8} \mu_{2} \omega_{1}-2 i \omega_{1} D_{1} C_{8}\right)}{\omega_{2}^{2}-\omega_{1}^{2}}, E_{32}=\frac{\hat{\lambda} C_{7}}{\omega_{2}^{2}} \text {, }
$$

$r_{11}=-\mu_{1}, r_{12}=+\frac{F}{2 \omega_{1}} \cos \left(\theta_{10}\right), r_{13}=\frac{\gamma}{2 \omega_{1}} \sin \left(\theta_{20}\right), r_{14}=\frac{\gamma}{2 \omega_{1}} b_{0} \cos \left(\theta_{20}\right)$,

$$
r_{21}=\left(\frac{\sigma_{1}}{a_{0}}-\frac{9 \alpha_{2} a_{0}}{8 \omega_{1}}-\frac{25 \alpha_{4} a_{0}^{3}}{16 \omega_{1}}\right), r_{22}=-\frac{F}{2 a_{0} \omega_{1}} \sin \left(\theta_{10}\right), r_{23}=\frac{\gamma}{2 \omega_{1} a_{0}} \cos \left(\theta_{20}\right) \text {, }
$$




$$
\begin{aligned}
& r_{24}=-\frac{\gamma}{2 \omega_{1} a_{0}} b_{0} \sin \left(\theta_{20}\right), r_{31}=-\frac{\lambda}{2 \omega_{2}} \sin \left(\theta_{20}\right), r_{32}=0, r_{33}=-\mu_{2}, r_{34}=-\frac{\lambda}{2 \omega_{2}} a_{0} \cos \left(\theta_{20}\right), \\
& r_{41}=\left(\frac{\sigma_{2}}{a_{0}}-\frac{9 \alpha_{2} a_{0}}{8 \omega_{1}}-\frac{25 \alpha_{4} a_{0}^{3}}{16 \omega_{1}}-\frac{\lambda}{\omega_{2} b_{0}} \cos \left(\theta_{20}\right)\right), r_{42}=-\frac{F}{2 \omega_{1} a_{0}} \sin \left(\theta_{10}\right), \\
& r_{43}=\left(\frac{\sigma_{2}}{b_{0}}+\frac{\gamma}{\omega_{1} a_{0}} \cos \left(\theta_{20}\right)+\frac{F}{2 \omega_{1} a_{0} b_{0}} \cos \left(\theta_{10}\right)-\frac{3 \alpha_{2} a_{0}^{2}}{8 b_{0} \omega_{1}}-\frac{5 \alpha_{4} a_{0}^{4}}{16 b_{0} \omega_{1}}\right), \\
& r_{44}=\left(\frac{\lambda}{2 \omega_{2} b_{0}} a_{0} \sin \left(\theta_{20}\right)-\frac{\gamma}{2 \omega_{1} a_{0}} b_{0} \sin \left(\theta_{20}\right)\right),
\end{aligned}
$$

\section{REFERENCES}

[1] Luo, A. C. J.: A mathematical modeling approach from nonlinear dynamics to complex systems. Nonlinear Systems and Complexity, 2019.

[2] A. Nayfeh, D. Mook, L. Marshall, Non-linear coupling of pitch and roll modes in ship motion, Journal of Hydronautics. 7 (4): 145-152, 1973.

[3] Kamel M., Kandil A., El-Ganaini W. A, and Eissa A. M., Active vibration control of a nonlinear magnetic levitation system via Nonlinear Saturation Controller (NSC), Nonlinear Dynamics, 77(3): 605-619, 2014.

[4] Abdelhafez H., Nassar M., Effects of time delay on an active vibration control of a forced and Self-excited nonlinear beam. Nonlinear Dynamics, 86 (1): 137-151, 2016.

[5] Bouchaala A., Nayfeh A. H, Younis M. I., Analytical study of the frequency shifts of micro and nano clamped-clamped beam resonators due to an added mass. Meccanica, 52(1-2): 333-348, 2017.

[6] EL-Sayed A.T., Bauomy H.S., Outcome of Special Vibration Controller Techniques Linked to a Cracked Beam. Applied Mathematical Modeling, 63: 266-287, 2018.

[7] Elnaggar, A.M., and Khalil, K.M.: The response of nonlinear controlled system under an external excitation via time delay state feedback. Journal of King Saud University - Engineering Sciences, 28(1): 75-83, 2014.

[8] Qian Y. J., Yang L.Y., Yang X.D., and Zhang W. Parametric stability analysis for planar bicircular restricted four-body problem. Astrodynamics, 2(2): 147-159, 2018.

[9] Kandil A., Eissa M., Kamel M., El-Ganaini W. and El-Gohary H. Actively controlling a rotating blade vibrations excited by a super harmonic force. Menoufia J. of Electronic Engineering Research (MJEER), $27(2) ; 2018$.

[10] Bouchaala A . Nayfeh A. H., and Younis M. I. Analytical study of the frequency shifts of micro and nano clamped-clamped beam resonators due to an added mass, Meccanica,52(1-2), 333-348, 2017.

[11] Abdelhafez H. M. and Nassar M. E. Suppression of Vibrations of a Forced and Self-excited Nonlinear Beam by Using Positive Position Feedback Controller PPF, British Journal of Mathematics \& Computer Science. 17(4): 1-19, 2016.

[12] Moeenfard H., Khadembashi M., Ghasemi A. H., and Baqersa J. Logic Analytical Modeling of a Piezoelectric Energy Harvesters under Random Base Excitation, A Conference and Exposition on Structural Dynamics, 9: 227-238, Apr2017.

[13] Omidi E., Mahmoodi S. N, and Shepard Jr. W. S., Multi positive feedback control method for active vibration suppression in flexible structures, Mechatronics, 33: 23-33, 2016.

[14] Omidi E.,and Mahmoodi S. N, Nonlinear integral resonant controller for vibration reduction in nonlinear systems, Acta Mechanica Sinica, 32(5): 925-934, 2016. 
[15] Meymand S. Z., Taheri M., Hosseinipour M., and Ahmadian M, Vibration Analysis of a Coupled Multibody Dynamic Model of a Contact Mechanics Roller Rig. Proceedings of the 2016 Joint Rail Conference JRC16 April 12-15, 2016, Columbia, SC, USA

[16] Omidi E.,and Mahmoodi S. Vibration Suppression of Distributed Parameter Flexible Structures by Integral Consensus Control. Journal of Sound and Vibration, 364: 1-13, 2016.

[17] El-Ganaini W.A., Saeed ·N.A., Eissa M., Positive position feedback (PPF) controller for suppression of nonlinear system vibration, Nonlinear Dynamics, 72(3): 517-537, 2013.

[18] Omidi E and Mahmoodi SN., Nonlinear vibration suppression of flexible structures using nonlinear modified positive position feedback approach. Nonlinear Dynamics, 79(2): 835-849, 2015.

[19] El-Sayed AT and Bauomy HS., Nonlinear analysis of vertical conveyor with positive position feedback (PPF) controllers. Nonlinear Dynamics, 83(1-2): 919-939, 2016.

[20] Eissa M. and Saeed N.A., Nonlinear vibration control of a horizontally supported Jeffcott-rotor system. Journal of Vibration and Control, 1-24, 2017.

[21] Abourabia A.M., Hassan K.M., and Selima E.S.: Painleve' analysis and new analytical solutions for compound KdV-Burgers equation with variable coefficients. Canadian Journal of Physics, 88(4), 211$221,2010$. 


\section{التحكم في اهتزازات منظومة غير خطية تحت تأثير قوة توافقية باستخدام التغذية المرتدة للموضع الايجابى}

\section{ياسر عبدالعزيز عامر 1 ، أشرف طه السيد ${ }^{2}$ ، عفاف أبو الفتوح صالح $3{ }^{3}$ ، أمل محم نصر ${ }^{3}$ ، هالة فايز سالمان، 2}

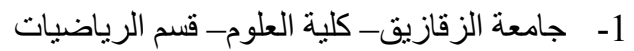

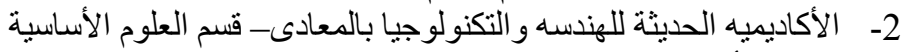

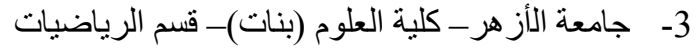

$$
\begin{aligned}
& \text { 4- جامعة القاهرة - كلية الحاسبات والمعلومات ـ قانسم العلوم الاساسية }
\end{aligned}
$$

في هذا البحث، تقليل الاهنزازات لمنظومة غير خطية مثارة بقوة نو افقية وذذلك عن طريق اضافة مخد الى

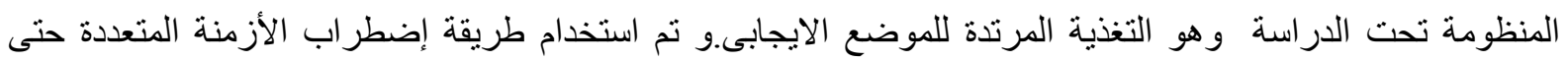
التقريب الثانى للحصول على الحل التقريبى للمعادلات التفاضلية التى تصف هذا النظام ,واستنتاج حالات الرنين المختلية ودر استها عدديا لتحديد أسوأ حالة رنين التى نم استنتاجها .تم الحصول على معادلات التجاوب لدراسة استقرار المنظومة

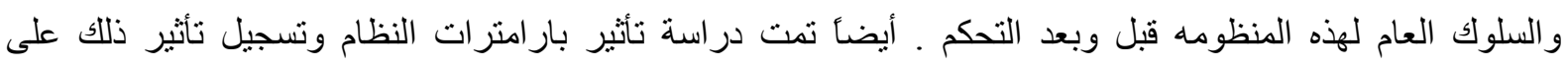

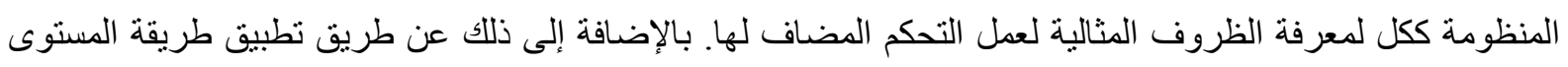
الطورى لدراسة استقرار النظام الاصلى بدون اضافة المخمد. وللتأكد من صحة الحلول التقربيية المستتجة بطريقة الأزمنة المتعددة تم عمل مقارنه بينها وبين الحلول العددية وذللك باستخدام طريقة رنج-كونا من الرتبة الرابعة وقد أبدت وند هذه المقارنة درجة تقارب جيدة جداً بينها ـ وأخير ا تم عمل مقارنة بين نتائج هذا البحث وما تم نشره فئ الابحاث السابقة. 